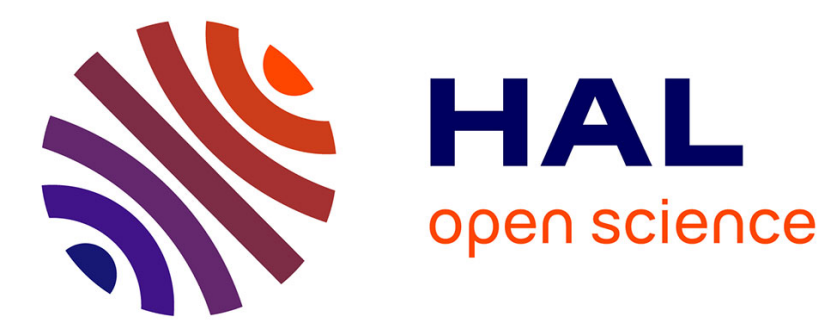

\title{
Motorcycle Dynamic Model Synthesis for Two Wheeled Driving Simulator
}

\author{
Salim Hima, Lamri Nehaoua, Nicolas Seguy, Hichem Arioui
}

\section{To cite this version:}

Salim Hima, Lamri Nehaoua, Nicolas Seguy, Hichem Arioui. Motorcycle Dynamic Model Synthesis for Two Wheeled Driving Simulator. 10th IEEE International Conference on Intelligent Transportation Systems (ITSC'07), 2007, Seattle, United States. pp.812-817. hal-00342938

\section{HAL Id: hal-00342938 \\ https://hal.science/hal-00342938}

Submitted on 1 Jul 2009

HAL is a multi-disciplinary open access archive for the deposit and dissemination of scientific research documents, whether they are published or not. The documents may come from teaching and research institutions in France or abroad, or from public or private research centers.
L'archive ouverte pluridisciplinaire HAL, est destinée au dépôt et à la diffusion de documents scientifiques de niveau recherche, publiés ou non, émanant des établissements d'enseignement et de recherche français ou étrangers, des laboratoires publics ou privés. 


\title{
Motorcycle Dynamic Model Synthesis for Two Wheeled Driving Simulator
}

\author{
Salim Hima, Lamri Nehaoua, Nicolas Séguy and Hichem Arioui
}

\begin{abstract}
This paper presents the development of motorcycle dynamics model. The considered vehicle contains six bodies linked with simple joints and parametrised by 11 degrees of freedom (DOF). The motorcycle model is to be used as a component in driving simulator application. It serves to investigate the influence of using a complete motorcycle dynamics model in inertial cues realism.

The choice of the modeling method is based on the algorithmic Lagrange equation representation. This method makes the implementation of the dynamic model very easy. In addition, the principal external forces affecting the motorcycle behavior are considered (pneumatic forces, driver actions, brakes ... etc). At the end of this article, some simulation results are presented in the case of street line motion.
\end{abstract}

Index Terms - motorcycle driving simulator, motorcycle dynamics, Lagrangian algorithmic formulation.

\section{INTRODUCTION}

Nowadays, motion-based simulators are become an important tools for different objectives in vehicles areas. They allow studding drivers-vehicles interaction, training a novice drivers and evaluation of a new assisted driving system in a safe environment. Initially, simulators are widely used in aeronautic industry and in automobile industry after for the objectives previously cited. Recently, few studies are accorded to transfer such tools to two wheeled vehicle [3], [5].

Two categories of simulators can be distinguished: a fixed base and dynamic base simulators. The first type is based solely on the visual and auditive cues while the second provides in addition, inertial cues for more realistic perception. One of the essential components of dynamic base simulators consists of vehicles dynamics model. In fact, motion base does move in limited workspace with respect to signals received from a vehicle dynamics model to feel the inertial forces by the drivers, i.e. accelerations or decelerations. Intuitively, the realism of the felt cues depends on the degree of accuracy and the validity envelope of this model. In the literature, the most of proposed models are developed for control purposes [11]. They are centered around a local motorcycle behaviors, and ignore some essential phenomena such as gyroscopic effect on the steering handlebar or pitching motion due to the suspension systems. These restrictions reduce the domain of validity of these models.

This work was supported by the National research agency (ANR) in SIMACIM framework

S. Hima, N. Séguy and H. Arioui are with IBISC FRECNRS 2873 Laboratory of Evry Val d'Essonne University, France 40 Rue de Pelvoux 91020 Evry Cedex France hima, nseguy, arioui@iup.univ-evry.fr

L. Nehaoua is with INRETS, 2 Av Gl Malleret-Joinville, 94114, Arceuil, France nehaouadinrets.fr
Recently, some advanced models have been proposed in literature. R. S. Sharp has used Autosim software to provide his model without any description on the applied method [12]. After, Cossalter et al have proposed a model based on Lagrange formalism and using natural coordinate to describe the configuration of motorcycle bodies. This method, for a complex systems with many DOFs, leads to a very complicated model, and so difficult to implement [2].

Deriving rigorous motorcycle dynamics, needs to use advanced mechanical techniques from multibody systems theory. Using absolute coordinates presents advantages so that the motion of each body in the multibody system is described using similar sets of generalized coordinates that do not depend on the topological structure of the system [2], [10]. The mass matrices of the bodies in the system have similar form and dimension, such that the computer program with simple structure can be developed based on the augmented formulation. A library of standard joint constraints can also be developed and used as a module in this computer program. However, this technique leads to a relatively complex numerical algorithm that must be used to solve the resulting mixed system of differential and algebraic equations DAE's [10].

Other alternative approaches for formulating the equations of motion of constrained mechanical systems are the recursive methods wherein these equations are formulated in terms of the joint degrees of freedom. This formulation leads to a minimum set of differential equations from which the constraint equations are automatically eliminated. The numerical procedure used in solving these differential equations is much simpler than the procedure used in the solution of the mixed systems of DAE's resulting from the use of the augmented formulation. In this paper, we have adopt this last formulation.

This article is organized into four sections. In the second section, a brief overview of our driving simulator platform is giving. The third section presents the dynamic model development of the motorcycle. Finally, Simulations results illustration and conclusions are done.

\section{SIMACOM SIMULATOR DESCRIPTION}

The driving simulator architecture, in SIMACOM project, is mainly conceived to feed the most important cues for rider's high fidelity immersion in simulation environment. It contains a visual and auditive systems allowing the rider immersion into a virtual traffic environment managed by ARCHISIM software [1], a motion-base platform to produce an inertial cues and a motion planner algorithm to splay 
the motion-base with a feasible and perceptual satisfactory reference trajectories, see Fig 1.

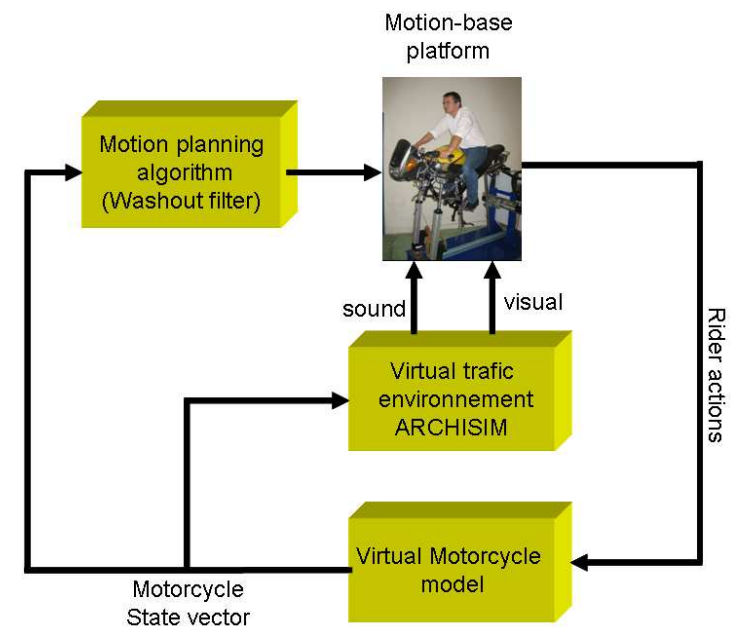

Fig. 1. Driving Simulator control architecture

Motion-base, see Fig 2, is essentially designed to reproduce the significant inertial effects during riding simulation. The mechanical design process was constrained by proposing a low cost platform with a suitable inertial cues felling. To achieve this objective, three degrees of freedom are privileged in this former simulator prototype. It's estimated that the roll, the pitch and the yaw motions are sufficient to make the rider in the most important scenarios such as acceleration, deceleration and cornering. The mobility of this platform has been calculated and shown to be equal to 3 directly controlled by 3 linear DC drives [8] .

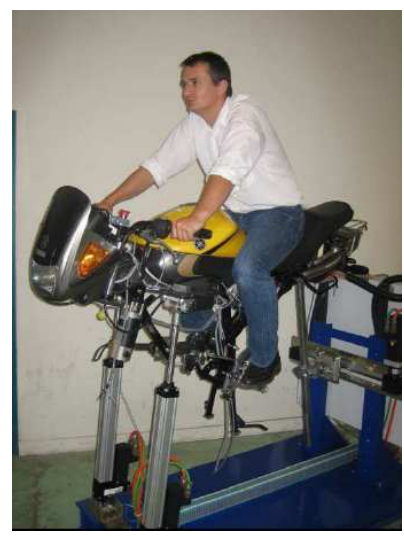

Fig. 2. Motion-base platform

Moreover, to increase the perception illusion, handlebar was endowed with double haptic feedback systems. The handlebar can move longitudinally with respect to a motorcycle saddle by means of a brushless servomotor. This movement aims to apply forces on the rider's arms, to reproduce partially the arms tension forces. In practice, these forces are caused by the inertial effect due to the inclination of rider's bust in the acceleration variation phases. In addition, the steering angle of the handlebar has been motorized in order to reproduce the effect of ground tire interaction forces and gyroscopic forces acting on the steering column, see Fig 3 . Besides, rider's actions are captured by placing different sensors on the motorcycle control organs (steering torque, throttle, brakes, clutch leavers and gearbox selector). For further technical details on the design and kinematic analysis of motion base, the reader can consult this reference [8].

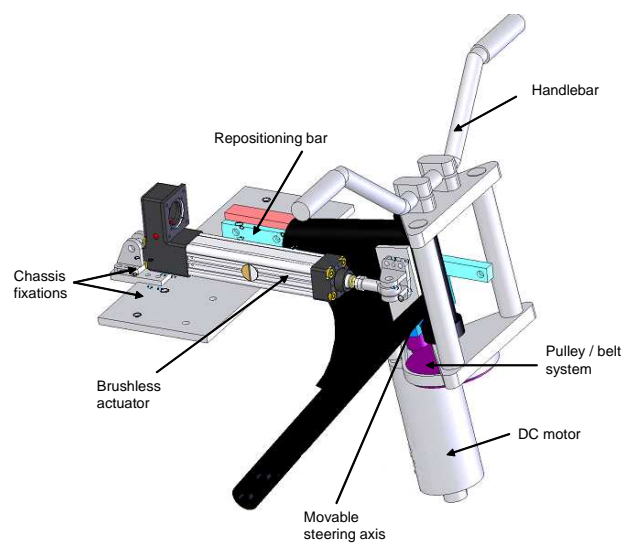

Fig. 3. Haptic feedback systems

When the rider act on the inputs organs, his actions are transmitted to the motorcycle dynamic model module to update the motorcycle state, see Fig 1. This allow to refresh the visual and auditive perceptual cues and splay the motion base platform with reference signals. Due to its workspace limitations, these signals can'nt be directly executable by the motion base platform. An appropriate adaptation is needed and can be made by way of motion planning module known by washout filter. In fact, Inputs of washout filter are motorcycle accelerations, velocities and orientations, while the outputs are the configuration commands to the motion base controllers [7].

At this stage of the development, we focus our effort to synthesize a dynamic model capturing the motorcycle behavior under the rider and environment interactions. The remainder of this paper will be devote to this aim.

\section{MotorCyCle DyNAMiCS}

Motorcycle dynamics module constitutes an interface between real and virtual worlds. It serves to update the visual subsystem by calculating the motorcycle configuration in the virtual environment and also indirectly control the motion base platform.

\section{A. Motorcycle configuration variables}

From a mechanical point of view, two wheels vehicles are multibody systems linked between them with a simple joints. In our case, we have considered 6 bodies: two tires, swinging arm, rear body (including the engine and the rider), upper front body and bottom front body. To derive the equations of motion of the motorcycle, we have applied the theory of multibody systems.

The configuration of the two wheeled vehicles, as depicted in Fig 4, is defined with respect to an inertial reference frame. 
It was parametrized by: the position $(x, y, z)$ of contact point between the rear tire and the road with respect to reference frame, the yaw and roll angles of the rear wheel $\psi$ and $\phi$, the swinging arm angle $\theta_{s}$, the rear (main) body angle $\theta$, the front suspension elongation $L$ and the rear and front wheels angle of rotation $\theta_{r}$ and $\theta_{f}$.

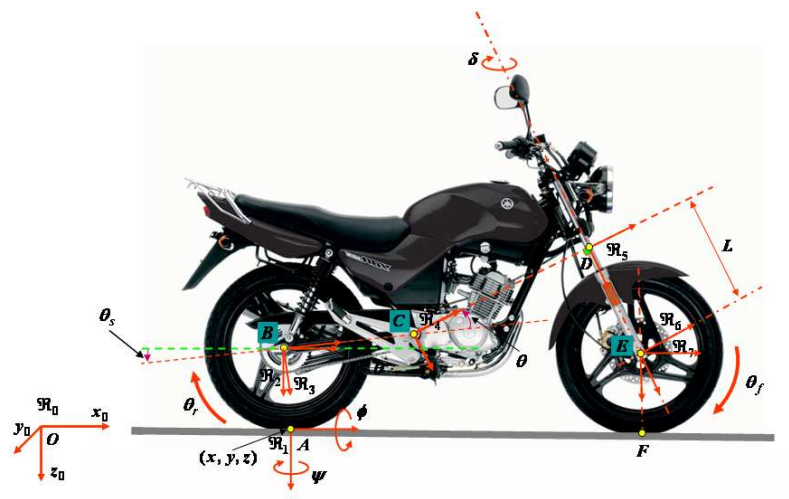

Fig. 4. Configuration variables definition

When these generalized coordinates are specified, the position and orientation of any motorcycle element can be defined accurately at any given time with respect to the inertial coordinate system.

\section{B. Motorcycle dynamics synthesis}

The equations of motion of the motorcycle are derived from Lagrange's equation formalism:

$$
\frac{\partial}{\partial t}\left(\frac{\partial \mathcal{L}}{\partial \dot{q}_{i}}\right)-\frac{\partial \mathcal{L}}{\partial q_{i}}=Q_{i}
$$

where $\mathcal{L}$ is the total energy of the bodies constituting the motorcycle. $q_{i}$ is the $i^{\text {th }}$ generalized coordinate and $Q_{i}$ are the generalized forces and moments vector corresponding to $q_{i}$. A forward application of this principle leads to a very complex equations of motion and consequently difficult to manipulate them. To overcome this problem, an algorithmic formulation of Lagrange's equation leading to the direct dynamic model and based on homogeneous matrix representation is adopted and can given by [4]:

$$
\begin{aligned}
& \sum_{j=i}^{n} \sum_{k=1}^{j}\left[\operatorname{tr}\left(\frac{\partial H_{j}}{\partial q_{i}} J_{j} \frac{\partial H_{j}^{T}}{\partial q_{k}}\right) \ddot{q}_{k}\right. \\
& \left.+\sum_{l=1}^{j} \operatorname{tr}\left(\frac{\partial H_{j}}{\partial q_{i}} J_{j} \frac{\partial^{2} H_{j}^{T}}{\partial q_{k} \partial q_{l}}\right) \dot{q}_{k} \dot{q}_{l}-m_{j} g^{T} \frac{\partial H_{j}}{\partial q_{i}} r_{j}\right]=Q_{i}
\end{aligned}
$$

${ }^{j} r_{j}$ is the center of mass position of the $j^{t h}$ body in its local coordinate frame. $m_{j}$ is the mass of the $j^{\text {th }}$ body. $g$ is the gravity vector. $t r$ is the trace operator of a matrix defined as $\operatorname{tr}(A)=\sum_{i=1}^{n} a_{i i} . H_{j}$ is the homogeneous matrix representing the position and the orientation of the body $j$ with respect to the inertial frame. Hence, the geometric relation between the global $P$ and local $P^{\prime}$ coordinates of the point belongs to the body $j$ is given by:

$$
P=H_{j} P^{\prime}
$$

where $P^{\prime}$ is a constant vector. $J_{j}$ is the matrix of inertia of the body $j$ expressed in the $j^{t h}$ body frame and given by:

$$
J_{j}=\left(\begin{array}{cccc}
\frac{-I_{x x}^{j}+I_{y y}^{j}+I_{z z}^{j}}{2} & I_{x y}^{j} & I_{x z}^{j} & m^{j} x_{c}^{j} \\
I_{y x}^{j} & \frac{I_{x x}^{j}-I_{y y}^{j}+I_{z z}^{j}}{2} & I_{y z}^{j} & m^{j} y_{c}^{j} \\
I_{z x}^{j} & I_{z y}^{j} & \frac{I_{x x}^{j}+I_{y y}^{j}-I_{z z}^{j}}{2} & m^{j} z_{c}^{j} \\
m^{j} x_{c}^{j} & m^{j} y_{c}^{j} & & m^{j} z_{c}^{j}
\end{array}\right.
$$

$I_{i}^{j}$ is the inertia tensor elements of the body $j$ in its local coordinates frame, $m_{j}$ is its mass and $\left(x_{c}^{j}, y_{c}^{j}, z_{c}^{j}\right)^{T}$ is a constant vector corresponding to the coordinates of its center of gravity expressed in its local coordinates frame.

\section{External forces}

In this section, we summarize the principal forces and moments affecting the motorcycle behavior.

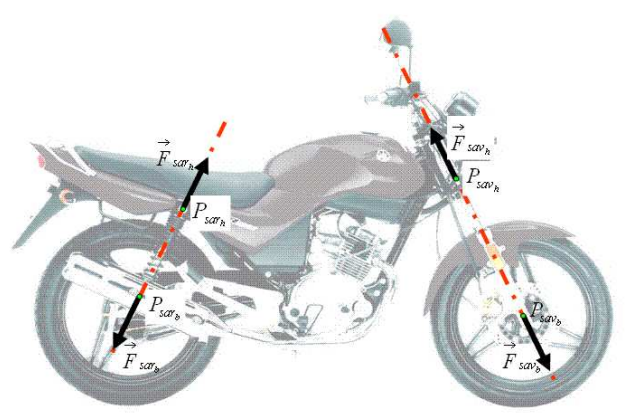

Fig. 5. Motorcycle suspension system configuration

1) Suspension forces: In order to absorb the shocks caused by the road irregularities and bumps, the motorcycle is equipped with a rear and front suspension systems. In this paper a linearly dependence of the forces generated by the suspensions on the elongations and their rates of change is considered:

$$
F_{i}=K_{i}\left(L_{i}-L_{i 0}\right)+C_{i} \dot{L}_{i}
$$

where $i=\{r s, f s\}$ designates the rear or front suspension, $L_{i 0}$ is its free load elongation, $K_{i}$ is the suspension stiffness and $C_{i}$ is the damper coefficient. So, the suspension length can be expressed as:

$$
L_{i}=\sqrt{\left(P_{i_{u}}-P_{i_{l}}\right)^{T}\left(P_{i_{u}}-P_{i_{l}}\right)}
$$

where $P_{i_{u}}$ and $P_{i_{l}}$ denote respectively the position of the upper and lower extremities of suspension $i$. Hence, the rate of change of $i^{t h}$ suspension length is given by:

$$
\dot{L}_{i}=\frac{1}{L_{i}}\left(\dot{P}_{i_{u}}-\dot{P}_{i_{l}}\right)^{T}\left(P_{i_{u}}-P_{i_{l}}\right)
$$

It is easy to express $\dot{P}_{i_{u}}$ and $\dot{P}_{i_{l}}$ in terms of partial derivatives of the configuration matrix of the $j^{\text {th }}$ body, at which they are attached:

$$
\begin{aligned}
\dot{P}_{m} & =\dot{H}_{j} P_{m}^{\prime} \\
& =\left(\sum_{n=1}^{j} \frac{\partial H_{j}}{\partial q_{n}} \dot{q}_{n}\right) P_{m}^{\prime}
\end{aligned}
$$


where $m=\left\{i_{u}, i_{l}\right\}$ and $P_{m}^{\prime}$ is a constant vector of local coordinates of the point $P_{m}$ expressed in local frame. Relation (8) shows that, $\dot{P}_{m}$ can be obtained by using quantities previously computed in equation (2).

2) Tire/road contact wrench: Behavior of the ground vehicles depends significantly on the nature of the interaction between the tire and the road. Indeed, in the last two decades, and due to the importance of this phenomena in the security of ground vehicles, several efforts are done to establish mathematical models characterizing the friction forces and moments of the contact tire/road. The well known and widely used one is Pacejka Magic formula. This model captures in steady-state motion, the tire/road forces and moments, in algebraic equations form, with respect to load and kinematic quantities, longitudinal slip $\kappa$ and lateral slip $\beta$. The steadystate behavior restriction still valid in the scope that, the effect of the tire dynamics is generally small compared to the effect of the complete motorcycle dynamics.

Initially, Pacejka model has been developed for four wheels vehicles. In this case, wheels steel approximately vertical and hence, the camber angle can be neglected. Other varieties of this model have been proposed and adapted to motorcycle tires by including the camber angle. This angle could achieves $50^{\circ}$ for racing motorcycles when cornering and consequently, could'nt be ignored [6].

Among a six components of the tire/road contact wrench, we have considered the principal ones: longitudinal and lateral forces and the aligning moment [13], [9]. Figures 6, 7 and 8 illustrate the shape of longitudinal and lateral forces and aligning moment vs longitudinal slip, for different values of lateral slip angle, corresponding to experimental identified data of the 160/70 tire type found in [13].

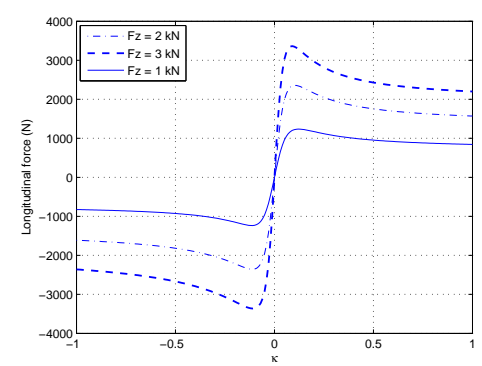

Fig. 6. Longitudinal force vs $\kappa$ for load force $F_{z}=1,2,3 \mathrm{kN}$

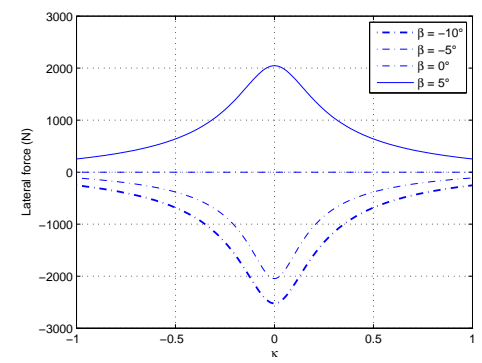

Fig. 7. Lateral force vs $\kappa$ for $\beta=5^{\circ}, 0^{\circ},-5^{\circ},-10^{\circ}$

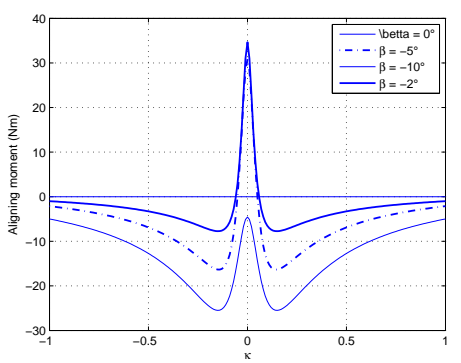

Fig. 8. Aligning moment vs $\kappa$ for $\beta=0^{\circ},-2^{\circ},-5^{\circ},-10^{\circ}$

3) Aerodynamic wrench: When the motorcycle moves, its interaction with the air generates an aerodynamic forces and moments. The most important components are the drag and the lift applied at the center of pressure, often located above the motorcycle center of gravity. The consequence of this deportation is the presence of an aerodynamic pitching moment. The drag force influences the maximum achievable velocity and also acceleration performance. The lift force can have an undesirable effects on the motorcycle performances. Indeed, it reduces the load on the wheels, and thus, decreases the tire adherence.

Mainly, the drag and the lift forces are expressed in a linear form with respect to the square of the motorcycle linear relative velocity with respect to the air:

$$
\begin{aligned}
F_{D} & =\frac{1}{2} C_{D} \rho S V_{a}^{2} \\
F_{L} & =\frac{1}{2} C_{L} \rho S V_{a}^{2}
\end{aligned}
$$

where $C_{D}$ and $C_{l}$ are dimensionless coefficient, $\rho$ is the air density, $V_{a}$ is motorcycle relative velocity and $S$ is the frontal motorcycle area exposed to the air flow.

4) Contact constraints forces: Keeping the tires in contact with the road imposes two constraints. In order to satisfy these constraints, we have placed two spring-dapper systems between the road and the tires contact points. This formulation can also capture tires carcasses elasticity features, as sketched in Fig 9.

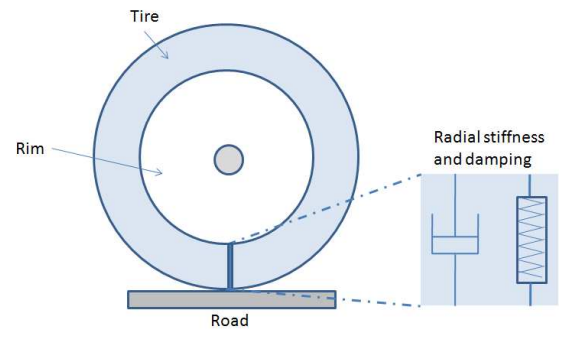

Fig. 9. Radial elasticity of the tire carcass

This formulation is more advantageous than using Lagrangian multipliers which need to include constraints stabilisers to prevent constraints divergence.

5) Input controls: The last considered external forces acting on the motorcycle behavior coming from the rider actions through the engine torque, brakes torques and finally 
steering torque. We have modeled these actions by a pure torque and we don't integrate their dynamic models, which will be considered in future works.

\section{SIMULATION RESULTS}

In the lake of presence of a motorcycle keeping up controller, we have limited the test of the developed motorcycle model to longitudinal behavior. For this aim, two test scenarios are considered, static and dynamic tests.

\section{A. Static test}

In this scenario, the motorcycle is at rest and a vertical square form force of amplitude $A=800 \mathrm{~N}$ has been applied on the saddle of the motorcycle, simulating a rider of $80 \mathrm{~kg}$ of weight as depicted in Fig 10. In this case, the rear and front suspensions are contracted when the force is applied and regain their initial elongations when the force is omitted, as illustrated in Fig 11 and Fig 13. Moreover, the main body pitch positively when the vertical force is applied, see Fig 12. This fact is a consequence of deportation of the application point of the test force, behind the center of mass of the main body. Fig 14 and Fig 15 illustrate the amount of violation of the rear and front contact constraints.

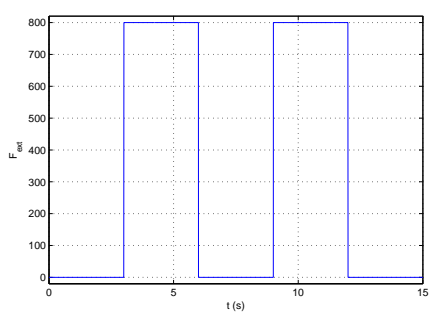

Fig. 10. Applied vertical square wave force

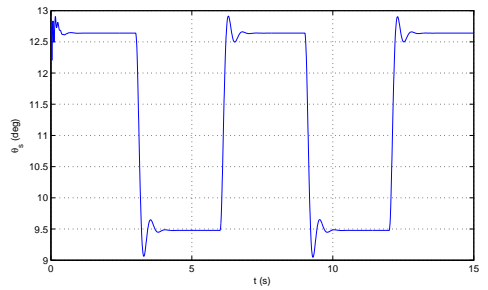

Fig. 11. Swinging arm angle evolution in the static test

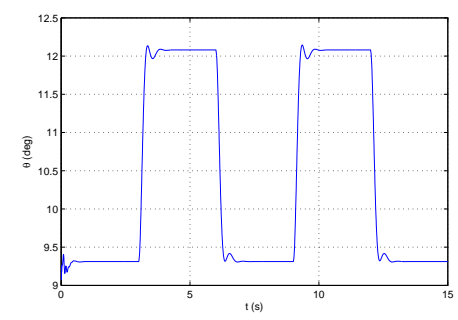

Fig. 12. Main body angle evolution in static test

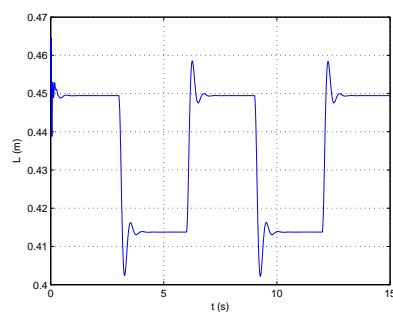

Fig. 13. Front suspension elongation evolution in static test

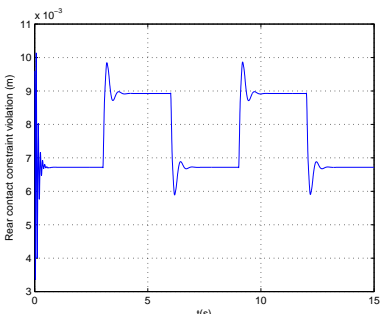

Fig. 14. Rear contact constraint violation

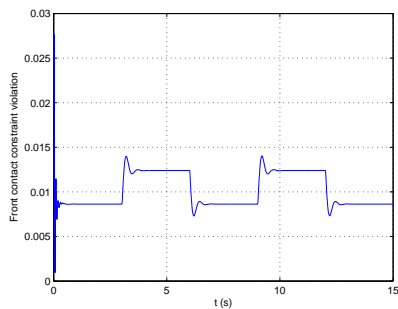

Fig. 15. Front contact constraint violation

\section{B. Dynamic test}

In this scenario, the motorcycle is subject to traction force of $500 \mathrm{~N}$ applied at the rear tire/road contact point. The shape of this force simulates the accelerated and unaccelerated phases as depicted in Fig 16. When the motorcycle accelerates, the inertia of the main body opposes to this motion by a force in the opposite direction. Consequently, the front suspension and also the rear suspension are contracted, and hence, the main body relative angle decreases, see Fig 19, Fig 17 and Fig 18. Fig 20 and Fig 21 depict, respectively, the violation of the rear and front contact constraints.

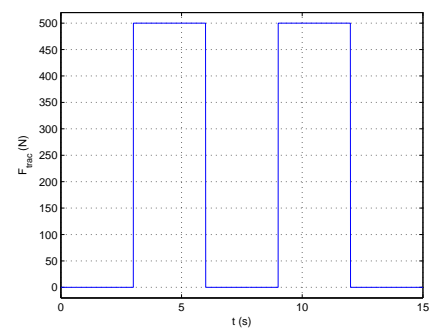

Fig. 16. Traction force shape in dynamic test 


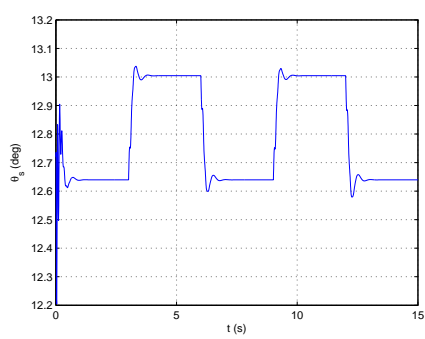

Fig. 17. Swinging arm angle evolution in the dynamic test

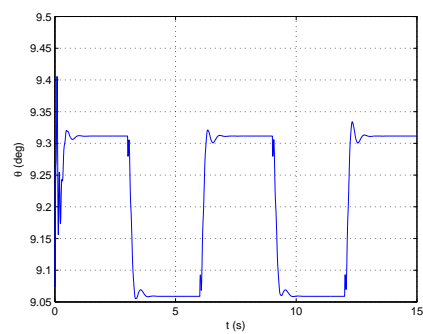

Fig. 18. Main body angle evolution in dynamic test

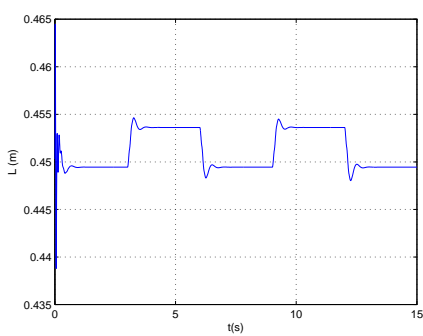

Fig. 19. Front suspension elongation evolution in dynamic test

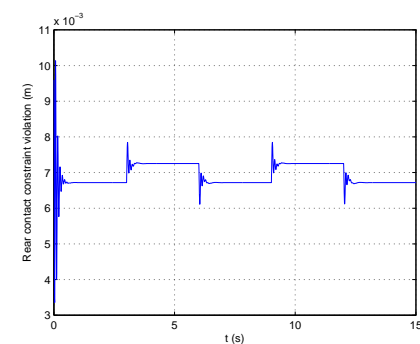

Fig. 20. Rear contact constraint violation

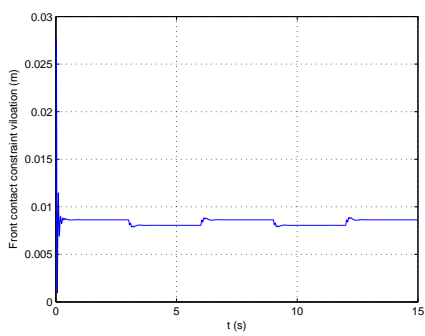

Fig. 21. Front contact constraint violation

\section{CONCLUSION AND FUTURE WORKS}

Based on Lagrange formulation, a motorcycle dynamic model dedicated to the driving simulator application is proposed. The development method is discussed and the advantages are outlined. In particular case, longitudinal motion, the simulation results confirm well a real behavior of the motorcycle model. As future works, a steering controller will be developed to test a very complex behavior of the synthesised model such as cornering. In addition, Comparison with the other approaches performances will be established. Besides, a more refined model will be proposed by considering the tire/road contact point migration in the tire circumference when cornering, motorcycle engine dynamics model, brakes dynamics model, and finally, a quantitative evaluation will be done for real-time applications.

\section{ACKNOWLEDGMENTS}

This work is supported by the National Research Agency (ANR) in SIMACOM framework.

\section{REFERENCES}

[1] A. Champion, S. Espie, and J. M. Auberlet, "Behavioral road traffic simulation with ARCHISIM," Summer Computer Simulation Conference, pp. 359-364, 1998.

[2] V. Cossalter and R. Lot, "A motorcycle multi-body model for real time simulations based on the natural coordinates approach," Vehicle System Dynamics, vol. 37, no. 6, pp. 423-447, 2002.

[3] D. Ferrazzini and al, "The moris motorcycle simulator: An overview," SAE, Tech. Rep., 2001.

[4] J. M. Hollerbach, "A recursive lagrangian formulation of manipulator dynamics," Massachusetts Institut of Technologiy : Artificial Intelligence Laboratory, Tech. Rep. 533, 1980.

[5] D.-S. Kwon and Al., "KAIST interactive bicycle simulator," IEEE - International Conference on Robotics and Automation, vol. 3, pp. 2313-2318, May 2001.

[6] R. Lot, "A motorcycle tire model for dynamic simulations: Theoretical and experimental aspects," Meccanica, an International Journal of Theoretical and Applied Mechanics, vol. 39, no. 3, pp. 207-220, 2004.

[7] L. Nehaoua, A. Amouri, and H. Arioui, "Classic and adaptive washout comparison for low cost driving simulator," IEEE Mediterrean Conference on Control and Automation, Lisboa, Portugal, 27-29 june, pp. 586-591, 2005.

[8] L. Nehaoua, S. Hima, N. Séguy, H. Arioui, and S. Espié, "Design of a new motorcycle riding simulator: Open-loop tests," American Control Conference, New York City, USA, july 11-13, 2007.

[9] H. B. Pacejka and I. J. M. Besselink, "Magic formulation tyre model with transient properties," Vehicle System Dynamics, vol. supplement 27, pp. 234-249, 1997.

[10] A. A. Shabana, Computational Dynamics. Johan Wiley and Sons, 1994

[11] R. S. Sharp, "The stability and control of motorcycles," Journal Mechanical Engineering Science, vol. 13, no. 5, pp. 316 - 326, 1971.

[12] — "Satbility, control and steering responses of motorcycles," Vehicle System Dynamics, vol. 35(4-5), pp. 291-318, 2001.

[13] R. S. Sharp, S. Evangelou, and D. J. Limebeer, "Advances in modelling of motorcycle dynamics," Multibody System Dynamics, vol. 12, pp. 251-283, 2004 